\title{
Magnetic fields of neutron stars in X-ray binaries
}

\author{
Mikhail Revnivtsev · Sandro Mereghetti
}

Received: date / Accepted: date

\begin{abstract}
A substantial fraction of the known neutron stars resides in X-ray binaries - systems in which one compact object accretes matter from a companion star. Neutron stars in X-ray binaries have magnetic fields among the highest found in the Universe, spanning at least the range from $\sim 10^{8}$ to several $10^{13} \mathrm{G}$. The magnetospheres around these neutron stars have a strong influence on the accretion process, which powers most of their emission. The magnetic field intensity and geometry, are among the main factors responsible for the large variety of spectral and timing properties observed in the X-ray energy range, making these objects unique laboratories to study the matter behavior and the radiation processes in magnetic fields unaccessible on Earth. In this paper we review the main observational aspects related to the presence of magnetic fields in neutron star X-ray binaries and some methods that are used to estimate their strength.
\end{abstract}

Keywords Neutron stars · Magnetic field · X-ray binaries

\section{Introduction}

X-ray binaries (XRBs) were the first astrophysical objects in which relativistic compact stars (neutron stars (NSs) and black holes) were detected. In 1962 the pioneering observations of the sky in the X-ray energy

M. Revnivtsev

Space Research Institute, Russian Academy of Sciences,

Profsoyuznaya 84/32, Moscow, Russia, 117997

E-mail: revnivtsev@iki.rssi.ru

S. Mereghetti

INAF, IASF-Milano, v. Bassini 15, I-20133 Milano, Italy

E-mail: sandro@iasf-milano.inaf.it range led to the discovery of the bright source Sco X1 (Giacconi et al. 1962), which is now known to contain an accreting NS. However, at that time the origin of the X-ray emission and the nature of the Sco $\mathrm{X}-1$ source were completely unknown. Thus, the first ones to recognize the existence of NSs were radio astronomers, who discovered the radio pulsars a few years later (Hewish et al. 1968; Gold 1968). Measurements of the spin-down of radio pulsars, interpreted as a loss of rotational energy due to the emission of magneto-dipole radiation, allowed the first estimates of their magnetic fields (e.g. Gunn \& Ostriker 1969). These were in agreement with earlier predictions based on the assumption that the magnetic flux of stars collapsing into NS is conserved, giving rise to NS magnetic fields as high as $B \sim 10^{12}-10^{16}$ G (Ginzburg 1964, Woltjer 1964).

The observations obtained by UHURU, the first satellite for X-ray astronomy, showed that many XRBs contain NSs with magnetic fields powerful enough to overcome the enormous gravitational drag of the NS and disrupt the flow of accreting matter, thus creating anisotropic emission patterns. These allowed rotating accreting NSs to be detected as regularly pulsating Xray sources through a lighthouse effect (Giacconi et al. 1971; Schreier et al. 1972, Tananbaum et al. 1972, Lamb et al. 1973, Davidson \& Ostriker 1973).

Since the time of these early discoveries, significant advances in observational astrophysics have provided an extremely rich dataset on NSs. Differences in their intrinsic properties (ages, magnetic fields, spin-periods, etc.), as well as in their environment (e.g., isolated or in binaries, density of interstellar medium, location in globular clusters, etc.), give rise to a variety of manifestations that can be studied in the whole electromagnetic spectrum. In this paper we concentrate on the NSs whose emission is mainly powered by accretion of mat- 
ter provided by a companion star. The accreting matter acts as a probe in the regions of our interest, in particular around magnetic NSs. Observations in X-rays (from $\sim 1 \mathrm{keV}$ to a few hundred $\mathrm{keV}$ ) are one of the most informative ways to explore the properties of NSs because this is the energy range where the NS surface and the matter in its immediate vicinity emit a significant part of their bolometric luminosity.

In Section 2 we introduce a few basic equations relevant for the physics of accretion and give a brief overview of the main properties of XRBs containing NSs. In the following Sections we focus on a few specific aspects closely related to the presence of magnetic fields: the observation of cyclotron lines (Sect. 3), and the interaction between the accretion flow and the NS magnetosphere (Sect. 45 , and 6).

\section{Accretion-powered neutron star X-ray binaries}

X-ray binaries are powered by accretion onto a compact object which gravitationally captures part of the mass lost by its companion star. The early evidence that many of the observed properties of XRBs depend on the type of companion star led to their classification into the two main classes of high mass X-ray binaries (HMXBs) and low mass X-ray binaries (LMXBs), based on the mass of the companion star. Extensive reviews of this vast subject can be found, e.g., in Lewin \& van der Klis (2010). Progress in observations led to the discovery of a large number of XRBs in our Galaxy and in the Magellanic Clouds (see, e.g., Liu et al. 2006, 2007, for recent catalogs of XRBs). At the moment, the most sensitive surveys of the whole Galactic plane obtained in hard X-rays have detected about two hundred sources, nearly equally divided between HMXBs and LMXBs (Krivonos et al. 2010, Bird et al. 2010, Baumgartner et al. 2013).

Before giving an overview of the main properties of XRBs containing NSs, we remind a few basic concepts and definitions that will be used in the following sections (see, e.g., Frank et al. 1992, for a more extensive discussion). We denote with $\mathrm{M}$ and $\mathrm{R}$ the NS mass and radius, and assume in all the numerical formulae the values $M=1.4 M_{\odot}$ and $R=12 \mathrm{~km}$. The NS moment of inertia is $I \approx 0.4 M R^{2}$, and its angular velocity is $\omega=2 \pi \nu=2 \pi / P$. The magnetic dipole moment is $\mu=B R^{3} / 2$, where $B$ is the field strength at the NS magnetic poles.

The accreting matter, on its way toward the compact object, releases a large amount of gravitational energy, heats up and emits mostly in the X-ray energy range. In the case of an accreting NS, the accretion luminosity is given by:

$L_{\mathrm{X}} \approx \frac{G M \dot{M}}{R} \approx 1.5 \times 10^{20} \dot{M} \operatorname{erg~s}^{-1}$,

where $\dot{M}$ is the mass accretion rate in $\mathrm{g} \mathrm{s}^{-1}$. This corresponds to a $\sim 17 \%$ efficiency of conversion of the rest mass energy of the accreting matter.

An upper limit to the accretion luminosity is given by the Eddington luminosity, $L_{\mathrm{Edd}}$, at which the radiation force equals the gravitational attraction, thus stopping the accretion flow:

$L_{\mathrm{Edd}}=\frac{4 \pi G M m_{\mathrm{p}} c}{\sigma_{\mathrm{T}}} \approx 1.3 \times 10^{38} \frac{M}{M_{\odot}} \operatorname{erg~s}^{-1}$,

( $c$ is the velocity of light, $m_{\mathrm{p}}$ is the proton mass, and $\sigma_{\mathrm{T}}$ is the Thompson cross section). The typical luminosities observed in bright XRBs are of the order of $\sim 10^{36}$ $10^{38} \mathrm{erg} \mathrm{s}^{-1}$. They imply accretion rates of $\sim 10^{16}-$ $10^{18} \mathrm{~g} \mathrm{~s}^{-1}$ (corresponding to $\sim 10^{-10}-10^{-8} M_{\odot} \mathrm{yr}^{-1}$ ). Much lower luminosities, down to $\sim 10^{31} \mathrm{erg} \mathrm{s}^{-1}$, are seen in the quiescent states of transient sources (note that other processes besides accretion might be at work to power these low luminosities).

To discuss the effects produced by the NS magnetic fields on the flow of accreting matter, hence on the $\mathrm{X}$ ray emission properties, it is useful to consider a few characteristic radii around the compact object. Their relative positions, which depend mainly on $\omega, \mu$, and density of the inflowing mass, determine the appearance of the NS (see, e.g., Lipunov et al. 1992, Campana et al. 1998: Bozzo et al. 2008).

The accretion radius, $r_{\mathrm{a}}$, defines the region in which the matter is gravitationally captured by the compact object. In the case of a NS accreting from a stellar wind with velocity $v_{\mathrm{w}}$, it is given by:

$r_{\mathrm{a}}=2 G M /\left(v_{\mathrm{w}}^{2}+v_{\mathrm{orb}}^{2}\right)$,

where $v_{\text {orb }}$ is the orbital velocity of the NS (usually negligible compared to the wind velocity $v_{\mathrm{w}}$ ).

The magnetic energy density, $B^{2} / 8 \pi$, around a magnetized NS rapidly rises towards the compact object (as $\propto r^{-6}$ in the case of a dipole and even faster in the case of higher multipole fields). A simple estimate of the distance from the NS where the magnetic pressure becomes equal to the ram pressure of the accretion flow gives the magnetospheric radius (see, e.g., Pringle \& Rees 1972; Lamb et al. 1973, Kluźniak \& Rappaport 2007):

$r_{\mathrm{m}}=\xi\left(\frac{\mu^{4}}{G M \dot{M}^{2}}\right)^{1 / 7}$,

where a dipolar magnetic field has been assumed. The parameter $\xi$ depends on the configuration of the flow: it 
is $\sim 1$ for a spherical inflow (Pringle \& Rees 1972), while it can be smaller, $\xi \sim 0.4$, in the case of a Keplerian disk (Ghosh \& Lamb 1979). Within $r_{\mathrm{m}}$ the motion of the matter is strongly influenced by the geometry of the magnetic field, which rotates rigidly with the star.

Two other relevant radii are the corotation radius, $r_{\mathrm{co}}=\left(G M / \omega^{2}\right)^{1 / 3}$, and the light-cylinder radius, $r_{\mathrm{lc}}=$ $c / \omega$, where the linear velocity of the rigidly corotating magnetosphere equals, respectively, the Keplerian velocity and the speed of light.

\subsection{High Mass X-ray Binaries}

HMXBs are systems in which the mass donor is typically an OB star with mass greater than 6-7 $M_{\odot}$. Known HMXBs have orbital periods from 0.2 days ( $\mathrm{Cyg}$ $\mathrm{X}-3$, with a Wolf-Rayet companion) to almost one year ( $P_{\text {orb }} \sim 262$ days of SAX J2239+6116) and spin periods ranging from $0.7 \mathrm{~s}$ (SMC X-1) up to $\sim 14 \mathrm{ks}(1 \mathrm{H} 1249$ $637)$. The detection of $\mathrm{X}$-ray pulsations in most of these systems was one of the first indications for the presence of magnetized NSs, with fields sufficiently strong $\left(\sim 10^{11-13} \mathrm{G}\right)$ to channel the accretion flow onto the magnetic poles and produce beamed X-ray emission. These magnetic fields are of the same order of those found in young radio pulsars, consistent with the evidence that HMXBs have ages smaller than few tens of Myrs, as implied by the presence of massive stars in these systems.

Extensive surveys of the Galaxy (e.g. Lutovinov et al. 2013) have revealed several tens of persistently bright HMXBs with luminosity $L_{\mathrm{X}} \sim 10^{36}-10^{37} \mathrm{erg}$ $\mathrm{s}^{-1}$ (positions of some brightest sources on the sky are shown in Fig. 1). Many of them are located in the spiral arms, associated to regions of recent star formation. Persistent HMXBs typically have OB supergiant companion stars, characterized by strong stellar winds with velocity $v_{\mathrm{w}}$ of the order of a thousand $\mathrm{km} \mathrm{s}^{-1}$ and mass loss rates $\dot{M}_{\mathrm{w}} \sim 10^{-7}-10^{-5} \quad M_{\odot} \mathrm{yr}^{-1}$. Such winds provide the matter which is accreted by the NS. Several HMXBs with OB companions are strongly absorbed in the soft X-ray energy range, due to the presence of dense circumstellar material, and could be discovered only recently thanks to observations in the hard X-ray range (Revnivtsev et al. 2003; Walter et al. 2003, $\mathrm{Ku}$ ulkers 2005).

SFXTs (Supergiant Fast X-ray Transients) have OB-type supergiant companions, like those of the persistent HMXBs, but they are characterized by strong $\mathrm{X}$-ray outbursts of short duration spanning a very large dynamic range - up to 3 or 5 orders of magnitude from quiescence to the peak of their outbursts (see, e.g., Sguera et al. 2006, Sidoli 2012). SFXTs spend most of the time at very low luminosities, and sporadically emit outbursts with duration of the order of few hours or even less (see e.g. reveiw in Paizis \& Sidoli 2014), although sometimes superimposed on longer periods of activity. Due to the rarity and short duration of their outbursts, SFXTs escaped detections until recently. Thanks to the extensive coverage of the Galactic plane with the INTEGRAL observatory, this class has now grown up to a dozen of sources (Romano et al. 2014). The mechanism at the basis of SFXT outbursts is not yet understood and several models have been proposed (e.g. in't Zand 2005; Sidoli et al. 2007; Walter \& Zurita Heras 2007; Grebenev \& Sunyaev 2007; Ducci et al. 2009).

The largest population of HMXBs consists of NSs with Be type companions (e.g. Reig 2011). Most of them are transient systems, which spend a large fraction of time in a low luminosity (or off-) state. Their outbursts are related to the presence of the dense equatorial outflows in the winds of Be stars, which are responsible for the $\mathrm{H}_{\alpha}$ emission lines seen in the optical spectra of these stars. The transient behavior of these XRBs occurs because the NS acquires a higher accretion rate when it crosses the Be equatorial disk and/or because the disk ejection itself is subject to long term variability.

Most NSs in HMXBs accrete matter gravitationally captured from the stellar winds of massive companions which underfill their Roche-lobe (e.g. Davidson \& Ostriker 1973). More rarely, the accretion flow is significantly modified by a Roche-lobe influence (e.g. Lamers et al. 1976).

The strong fields of NSs channel the accreting matter onto the magnetic poles, forming an accretion column (e.g. Basko \& Sunyaev 1976, Lyubarskii \& Sunyaev 1988, Mushtukov et al. 2014). Most of the kinetic energy of the matter falling onto the NS surface should be released as radiation of the accretion column, with a luminosity of the order of $\sim 0.2 \dot{M} c^{2}$ (eq. (1)). The free fall velocity near the NS surface is $\sim 0.6 c$, which corresponds to effective temperatures larger than $10^{12} \mathrm{~K}$ (>100 MeV). The observed emission, however, is more concentrated in the 1-20 keV energy band and the spectrum is significantly different from that of a simple blackbody emission - the spectrum is typically a power law $\left(d N / d E \propto E^{-\Gamma}\right)$ with photon index $\Gamma \sim 1$ up to energies 10-30 keV and an exponential cutoff at higher energies (see, e.g., White et al. 1983, Nagase 1989 Filippova et al. 2005: Caballero \& Wilms 2012). The emission pattern is strongly anisotropic, giving rise to easily detectable X-ray pulsations due to the rotation of the NSs. In most sources the pulse profiles show significant variations as a function of energy, luminosity level, orbital phase, and time. 


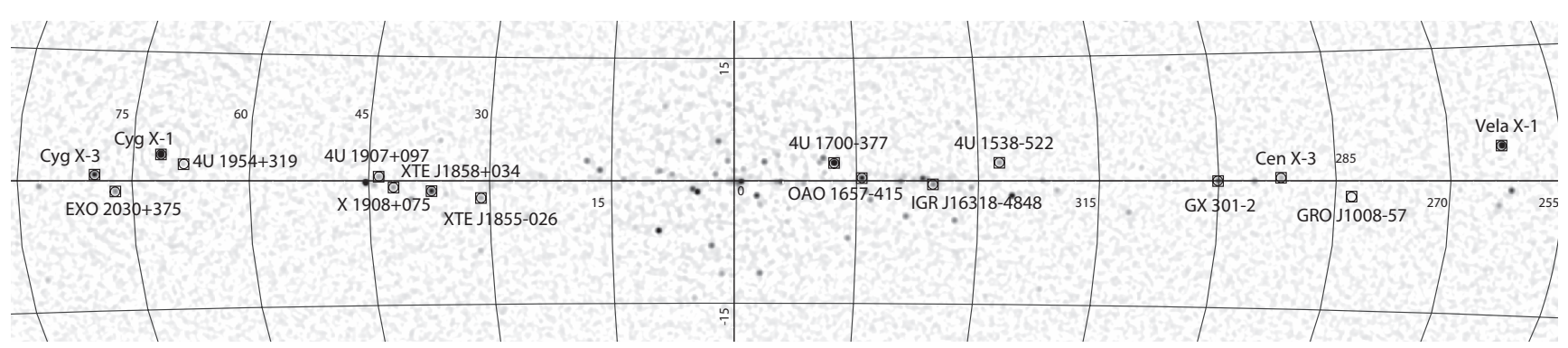

Fig. 1 Image of the central region of the Galactic plane (in Galactic coordinates), obtained by the INTEGRAL observatory in the energy band 17-60 keV. The positions of some of the brightest HMXBs in our Galaxy are indicated. The vast majority of them contain accreting magnetic NSs.

The details of the spectral formation in HMXBs are not yet fully understood, in spite of very serious efforts in this direction (Nagel 1981; Lyubarskii \& Sunyaev 1982, Meszaros \& Nagel 1985; Burnard et al. 1991; Shibanov et al. 1992; Klein et al. 1996; Becker \& Wolff 2005, 2007). Modelling the spectra of the accretion column on NS surfaces of X-ray pulsars is complicated by: a) the presence of powerful radiation pressure, which determines the dynamics of the settling flow, b) the strong magnetic field, which modifies all scattering cross sections and thus the radiation transfer, and c) the presence of fast bulk motion of the infalling matter, which contributes to the Compton upscattering of the outgoing radiation. All these complications should be solved self-consistently.

\subsection{Low Mass X-ray Binaries}

The companions of NSs in LMXBs are typically late type dwarf stars with mass below 1-2 $M_{\odot}$. Most LMXBs have orbital periods shorter than one day. The companion stars fill their Roche-lobe and accretion proceeds with the formation of a disk around the compact object. LMXB can have ages up to a few Gyrs, as reflected by their association with the old stellar population in the Galactic bulge and by their presence in globular clusters. The existence of dynamically important magnetic fields in LMXBs, able to channel the accretion flow onto the NS magnetic poles, has been under discussion for a long time because regular pulsations were originally seen only in a few peculiar members of this class, like Her X-1 $(P=1.24 \mathrm{~s}), 4 \mathrm{U}$ 1626-67 ( $P=7.7$ s) and GX $1+4(P=130$ s) (see Liu et al. 2007, and ref. therein). Extensive searches for pulsations in other bright LMXBs were unsuccessful (see, e.g., Mereghetti \& Grindlay 1987; Vaughan et al. 1994) and the presence of NSs, rather than accreting black holes, in these systems could only be established through the observation of type I X-ray bursts - thermonuclear explosions in the accreted matter which accumulates onto the NS surface (see, e.g., reviews in Lewin et al. 1993: Cumming 2004).

Thanks to the large effective area provided by the instruments on the RXTE satellite, X-ray pulsations were finally discovered in LMXBs (Wijnands \& van der Klis 1998). At the moment we know 15 systems of this class in which the NSs create persistent or intermittent $\mathrm{X}$-ray pulsations with periods of a few milliseconds (see, e.g., Patruno \& Watts 2012) and several sources which show millisecond pulsations during type I bursts (e.g. Strohmayer 2001). These findings show that the NSs in LMXBs have spin periods much shorter than those in HMXBs. Their fast rotation is due to the spin-up torque applied on the NS by the rapidly rotating Keplerian accretion disk, which extends close to the star surface. This implies magnetic fields $\mathrm{B} \sim 10^{8-10} \mathrm{G}$, smaller than those found in HMXBs, which could be a consequence of the old age of the NSs in LMXBs, possibly coupled to the field decay induced by the accretion process (e.g. Taam \& van den Heuvel 1986, Harding \& Lai 2006). Such low magnetic fields are at the basis of the well established recycling scenario, which explains the millisecond radio pulsars as old NSs spun-up by the accretion process in LMXBs (Bisnovatyi-Kogan \& Komberg 1976: Bhattacharya \& van den Heuvel 1991; Srinivasan 2010).

The weak magnetic field of these NSs is also at the basis of the different X-ray spectral properties of LMXBs and HMXBs. In general, at energies below $\sim 20$ $\mathrm{keV}$ the spectra of LMXBs are much softer than those of HMXBs. This is due to the fact that a large fraction of the observed X-rays originates in optically thick accretion disks, which in LMXBs extend close the compact object where they reach temperatures of only a few $\mathrm{keV}$. 


\section{Resonant cyclotron features in the X-ray spectra of accreting NSs}

The energy spectra of several accreting magnetic NSs show the presence of absorption features at energies of tens of keV. Discovered more than thirty years ago (Truemper et al. 1978; Wheaton et al. 1979), they were already expected by theoreticians (Gnedin \& Sunyaev 1974). These features were immediately interpreted as due to cyclotron resonant scattering of the outgoing radiation by electrons in a strong magnetic field and provided the first direct evidence for magnetic fields of the order of $10^{12-13} \mathrm{G}$ in the line-forming regions. The line energies are determined by the separation of the quantized Landau energy levels of electrons, which, in a magnetic field of strength $B$, is given by:

$E_{\mathrm{c}}=\hbar \frac{e B}{m_{e} c}=11.6 \frac{B}{10^{12} \mathrm{G}} \mathrm{keV}$

Due to the gravitational redshift $z$ at the position where the scattering occurs, the lines in the observer's frame of reference appear at $E_{\mathrm{c}} /(1+z)$.

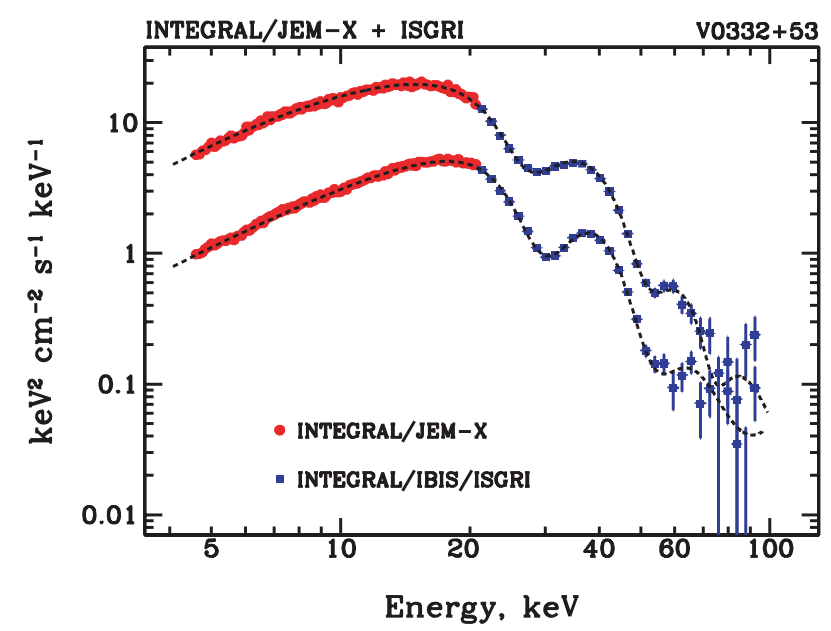

Fig. 2 Energy spectrum of the accretion powered pulsar $\mathrm{V} 0332+53$ at two intensity levels measured with the INTEGRAL observatory. At least two cyclotron absorption lines are clearly visible (from Tsygankov et al. 2006).

Over the last decades, cyclotron absorption features have been detected from more than twenty sources (see Table 1). In a few sources one or more harmonics of the fundamental line are also seen (Santangelo et al. 1999; Tsygankov et al. 2006). One of the best examples is presented in Fig.2. Note that the line energies given in Table 1 are only approximate values, since many sources show line energy variations as a function of the spin phase, time, and/or flux. In addition, the exact values of the line parameters derived from the spectral fits depend on the spectral model used to describe the continuum. Despite these uncertainties, it is clear that the observed line energies imply magnetic fields in the range $\sim 10^{12}-10^{13} \mathrm{G}$, both for NSs with Be and supergiant (SG) companions. Note that Her X-1 and GX $1+4$, the only LMXB showing cyclotron lines, are not representative members of the LMXB population: they resulted from different evolutionary histories and have magnetic fields similar to those of HMXBs. Among the most recent discoveries, it is worth noting that the first cyclotron line discovered in a member of the SFXT class indicates a "standard" field value (Bhalerao et al. 2014). This does not support the models invoking strong magnetic fields to explain these sources (e.g. Bozzo et al. 2008).

Variations of the line properties as a function of the source luminosity are particularly interesting since, in principle, they offer the possibility to study how the geometry of the accretion region changes at different mass accretion rates. In the simplest interpretation, the observed variations of line energy, $E_{\mathrm{c}}$, as a function of the luminosity, $L_{\mathrm{X}}$, trace the height of the Xray emitting shock in the accretion column. At luminosities above the local critical value $\left(L_{\mathrm{X}} \gtrsim 10^{37} \mathrm{erg}\right.$ $\left.\mathrm{s}^{-1}\right)$, as the mass accretion rate increases the radiation pressure moves the shock farther from the NS surface, where the magnetic field is weaker. Hence an anticorrelation between $E_{\mathrm{c}}$ and $L_{\mathrm{X}}$ is expected (Basko \& Sunyaev 1976, Burnard et al. 1991). This behavior has been observed in V0332+53 (Tsygankov et al. 2006) and in 4U 0115+63 (Nakajima et al. 2006). However, the analysis of another outburst from the latter source indicates that the reported anticorrelation might be an artifact caused by an improper modelling of the underlying variations of the continuum spectrum (Müller et al. 2013b). A positive correlation between $E_{\mathrm{c}}$ and $L_{\mathrm{X}}$ has instead been seen in Her X-1 (Staubert et al. 2007) and in a few other sources (see Table 1). Their different behavior has been attributed to the lower luminosity of these sources, in which gas pressure and Coulomb scattering become important in shaping the geometry and emission pattern of the accretion column (Becker et al. 2012).

There is general agreement that the observed lines are due to resonant electron scattering in a strong magnetic field and models based on the transmission of radiation through a highly magnetized medium in the accretion column can account for most of the observations (see e.g. Schönherr et al. 2007, Nishimura 2008). However, the simple picture outlined above predicts a luminosity-dependence of $E_{\mathrm{c}}$ at high accretion rates stronger than the observed one (typically $E_{\mathrm{c}}$ varies by less than 20-30\% when the luminosity changes by an order of magnitude). A recent model to overcome these 
Table 1 XRBs with cyclotron resonance features (updated from Caballero \& Wilms 2012). The $5^{\text {th }}$ coumn gives the number of harmonics. The + and - symbols in the $6^{t h}$ column indicate a positive or negative correlation between $E_{\mathrm{c}}$ and $L_{\mathrm{X}}$ (tentative reporst are indicated by a question mark); the $=$ symbol indicates that $E_{\mathrm{c}}$ has been seen to stay constant during luminosity changes.

\begin{tabular}{|c|c|c|c|c|c|c|}
\hline Name & Type & $\begin{array}{l}E_{\text {cyc }} \\
\mathrm{keV}\end{array}$ & $\begin{array}{c}\mathrm{B} /(1+\mathrm{z}) \\
10^{12} \mathrm{G}\end{array}$ & $\begin{array}{l}\text { Num. } \\
\text { harm. }\end{array}$ & $\begin{array}{l}E_{\mathrm{c}}-L_{\mathrm{X}} \\
\text { correl. }\end{array}$ & References \\
\hline Swift J1626.6-5156 & $\mathrm{Be}$ & 10 & 0.9 & 1 & + & DeCesar et al. $(\overline{2013})$ \\
\hline $4 \mathrm{U} 0115+63$ & $\mathrm{Be}$ & 11 & 1 & 4 & $-?$ & Wheaton et al. $(1979)$; Müller et al. (2013b) \\
\hline KS $1947+300$ & $\mathrm{Be}$ & 12 & 1 & & & Fürst et al. (2014a) \\
\hline IGR J17544-2619 & SFXT & 17 & 1.5 & & & Bhalerao et al. (2014) \\
\hline $4 \mathrm{U} 1907+09$ & SG & 18 & 1.5 & 1 & & Makishima \& Mihara (1992); Cusumano et al. (1998) \\
\hline $4 \mathrm{U} 1538-52$ & SG & 23 & 2 & & + & Clark et al. (1990); Hemphill et al. $(2014)$ \\
\hline Vela X-1 & SG & 25 & 2.1 & 1 & + & Kendziorra et al. (1992); Fürst et al. (2014b) \\
\hline V $0332+53$ & $\mathrm{Be}$ & 26 & 2.2 & 2 & - & Makishima et al. $(\overline{1990}) ;$ Tsygankov et al. $(2010)$ \\
\hline X Per & $\mathrm{Be}$ & $29 ?$ & 2.5 & & & Coburn et al. (2001); Doroshenko et al. (2012b) \\
\hline Cen X-3 & SG & 30 & 2.6 & & & Santangelo et al. (1998); Suchy et al. $(2008)$ \\
\hline Cep X-4 & $\mathrm{Be}$ & 31 & 2.7 & & & Mihara et al. (1991); McBride et al. (2007) \\
\hline RX J0520.5-6932 & $\mathrm{Be}$ & 32 & 2.8 & & & Tendulkar et al. (2014) \\
\hline RX J0440.9+4431 & $\mathrm{Be}$ & 32 & 2.8 & & & Tsygankov et al. (2012) \\
\hline IGR J16493-4348 & SG & 32 & 2.8 & & & D'Aì et al. (2011) \\
\hline MXB 0656-072 & $\mathrm{Be}$ & 33 & 2.8 & & & Heindl et al. (2003); McBride et al. (2006) \\
\hline GX 301-2 & SG & 35 & 3 & & & Makishima \& Mihara (1992); Kreykenbohm et al. (2004) \\
\hline XTE J1946+274 & $\mathrm{Be}$ & 36 & 3.1 & & $+?$ & Heindl et al. (2001); Müller et al. (2012) \\
\hline $4 \mathrm{U} 1626-67$ & LMXB & 37 & 3.2 & & $=$ & Orlandini et al. (1998); Camero-Arranz et al. (2012b) \\
\hline Her X-1 & LMXB & 41 & 3.5 & & + & Truemper et al. (1978); Staubert et al. (2007) \\
\hline A $0535+26$ & $\mathrm{Be}$ & 46 & 4 & 1 & $=$ & Kendziorra et al. (1992); Müller et al. (2013a) \\
\hline GX 304-1 & $\mathrm{Be}$ & 51 & 4.4 & & + & Yamamoto et al. (2011); Klochkov et al. (2012) \\
\hline 1A $1118-61$ & $\mathrm{Be}$ & 55 & 4.7 & & & Doroshenko et al. (2010b); Maitra et al. (2012) \\
\hline GRO J1008-57 & $\mathrm{Be}$ & 78 & 6.7 & & & Shrader et al. $(\overline{1999) ;}$ Bellm et al. $(2014)$ \\
\hline
\end{tabular}

problems is presented by Nishimura (2014), while a different explanation is based on the idea that the cyclotron lines are formed as a result of reflection of the accretion column X-ray emission from magnetized matter on the NS surface (Poutanen et al. 2013).

\section{Interaction between NS magnetosphere and accretion flow}

Although the details of the interaction between the rotating magnetosphere and the accretion flow are quite complicated and not yet fully understood (see, e.g., $\mathrm{Uz}-$ densky 2004, Lai 2014), it is generally assumed that accretion onto the NS surface can occur if the magnetospheric radius (eq. (4)) is smaller than the corotation radius. If, instead, $r_{\mathrm{m}}>r_{\mathrm{co}}$, the centrifugal barrier should stop the flow and inhibit continuous accretion (Illarionov \& Sunyaev 1975, Stella et al. 1986). In this case, non-stationary accretion might still proceed as the matter piles up around the intrinsically unstable magnetospheric boundary. Depending on its specific angular momentum, the captured plasma may form a quasispherical shell (Pringle \& Rees 1972, Ikhsanov 2007; Shakura et al. 2012) or reside in a so-called "dead accretion disk" (Sunyaev \& Shakura 1977; Baan 1979; D'Angelo \& Spruit 2010, 2012).
Accretion can proceed in two ways: either quasispherically or via a geometrically-thin disk. The latter scenario typically occurs when the mass donor star fills its Roche-lobe, as it happens in most LMXBs. In the case of wind-accreting HMXBs, quasi-spherical accretion can occur if the angular momentum of the mass captured within the accretion radius is sufficiently small. This can be evaluated by considering the circularization radius, i.e. the minimum distance from the NS which the accreting matter can reach without loss of angular momentum. The circularization radius, $r_{\text {circ }}$, can be roughly estimated by equating the specific angular momentum of the captured stellar wind matter to that on a Keplerian orbit (see, e.g., Frank et al. 1992):

$j \sim \frac{\pi r_{\mathrm{a}}^{2}}{2 P_{\text {orb }}} \sim \sqrt{G M r_{\text {circ }}}$,

This leads to:

$r_{\text {circ }} \sim \frac{4 \pi^{2} G^{3} M^{3}}{P_{\mathrm{orb}}^{2} v_{\mathrm{w}}^{8}}$

$r_{\text {circ }} \sim 3.3 \times 10^{6}\left(\frac{1 \text { day }}{P_{\text {orb }}}\right)^{2}\left(\frac{1000 \mathrm{~km} \mathrm{~s}^{-1}}{v_{\mathrm{w}}}\right)^{8} \mathrm{~cm}$

For typical values of NS magnetic fields, mass accretion rates, and stellar wind velocities of young OB-type stars, it turns out that, for wide binaries $\left(P_{\text {orb }}>\right.$ a few 

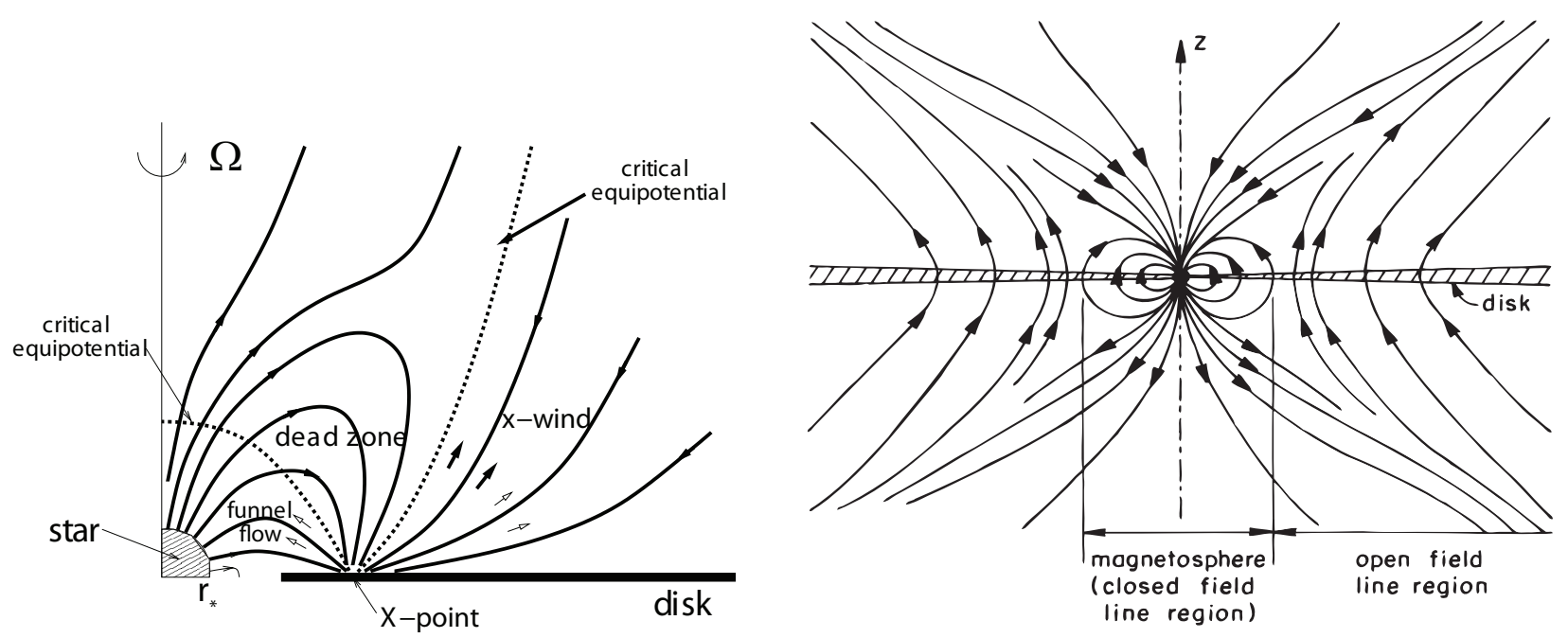

Fig. 3 Models of magnetosphere-accretion disk interaction of Shu et al. (1994) (left panel) and Lovelace et al. (1995) (right panel). Both models imply that the accretion disk matter is frozen into the NS magnetic field lines only over a relatively small transition region.

days), a disk cannot form because $r_{\text {circ }}<r_{\mathrm{m}}$. In this case the accreting matter should settle onto the rotating magnetosphere in some kind of a quasi-spherical flow.

The resulting interaction is a long standing problem and still a matter of debate (see, e.g., Arons \& Lea 1976: Lamb et al. 1977; Davies et al. 1979, Illarionov \& Kompaneets 1990; Ziolkowski 1985, Bozzo et al. 2008; Shakura et al. 2012, 2013). Unsettled questions on details of the magnetosphere-flow interaction sometimes lead to controversial suggestions about the presence of utrastrong magnetic fields in pulsars with long spin periods (see e.g. Finger et al. 2010, Doroshenko et al. 2010a Reig et al. 2012, Ho et al. 2014). The root of this problem is in the assumptions on the values of the torques exerted by the accreting matter settling to the NS magnetosphere.

Contrary to the disk accretion case, where the torques are relatively well understood and the predicted scalings confirmed by observations (see Sect. 4), the issue of the net torque acting on the NS is much less clear in the case of quasi-spherical accretion (see discussion of this problem in Shakura et al. 2012, 2013, Postnov et al. 2014).

In the cases of accretion from a companion star in tight binaries (where Roche-lobe overflow occurs), from the equatorial disks around Be stars, and from low mass companions, the specific angular momentum of the gravitationally-captured matter can be sufficiently large to form a disk before reaching the NS magnetosphere.
Some early works (e.g. Ghosh \& Lamb 1978, 1979) assumed that the NS magnetic field can penetrate the disk over a large range of radii, forming a relatively wide transition zone. It was later shown that this scenario requires an unphysically high magnetic diffusivity in the accretion disk (see, e.g., Wang 1995, Lesur \& Longaretti 2009). Calculations assuming a more realistic magnetic diffusivity indicate that the NS magnetic field can penetrate the disk only over a quite small range of radii (Campbell 1992; Shu et al. 1994, Lovelace et al. 1995), comparable to the thermal disk scale height $h \sim c_{s} / \omega_{\mathrm{K}}$, where $c_{s}$ is the sound speed in the disk and $\omega_{\mathrm{K}}$ is the Keplerian frequency. In these models the inner part of the flow is frozen into the closed field lines magnetosphere, and the outer part of the flow contains open field lines leading to the possibility of magnetically driven outflows (see Fig 3 .

The thickness of this transition region influences the structure of the accretion column onto the NS. If the area of the footprint of the accretion flow is small, the release of energy brought by the flow can be locally super-Eddington and can create a radiative shock which stops the infalling plasma. Despite significant theoretical efforts (e.g. Basko \& Sunyaev 1976, Ghosh \& Lamb 1978, 1979, Campbell 1992; Shu et al. 1994; Lovelace et al. 1995) the width of the transition region remains uncertain, and until recently could not be estimated observationally. A method to estimate the thickness of the transition region based on measurements of the cooling time of matter in the accretion column has been proposed for magnetic white dwarfs (Semena \& Revnivtsev 
2012 Semena et al. 2014). The inferred plasma penetration depth at the boundary of the magnetosphere is in general agreement with earlier theoretical (Shu et al. 1994 Lovelace et al. 1995). Extending these results to accreting magnetic NSs indicates that the footprint of their accretion columns might occupy an area as small as $<10^{-6}$ of the total NS surface.

\subsection{Spin-period variations}

Matter that moves toward the NS carries angular momentum, which can be added to that of the NS. If the accretion occurs via a Keplerian disk, the spin-up torque acting on the NS can be estimated as $K_{\mathrm{su}} \approx$ $\dot{M} \sqrt{G M r_{\mathrm{m}}}$ (Pringle \& Rees 1972). On the other hand, the rotating magnetosphere can lose angular momentum via different mechanisms. For example, it can transfer angular momentum via large scale magnetic field threading the external regions of the accretion disk, which have a slower rotation (e.g. Ghosh \& Lamb 1979. Lovelace et al. 1995, Wang 1995), or it can lose angular momentum by some matter outflow (e.g. Illarionov \& Kompaneets 1990). The spin-down torque $K_{\text {sd }}$ acting on the NS depends on how the accretion in the binary occurs (e.g. via Keplerian disk or via quasi-spherical flow) and is still a matter of debate (see e.g. Davidson \& Ostriker 1973, Kundt 1976, Lipunov 1981, Bisnovatyi-Kogan 1991; Wang 1995; Li \& Wang 1996 Kluźniak \& Rappaport 2007: Shakura et al. 2012). Leaving aside these complications, we can write the evolution of the spin period of NS accreting from a disk in a generalized form:

$I \frac{d \omega}{d t}=k_{\mathrm{m}} \dot{M} \sqrt{G M r_{\mathrm{m}}}-K_{\mathrm{sd}}$

where $k_{\mathrm{m}}$ is a numerical constant of the order of unity.

If the spin-up torque is significantly higher than the spin-down torque (e.g. during episodes of high mass accretion rate), the dependence of the spin-up rate on the source luminosity/mass accretion rate can be expressed as follows:

$\dot{\nu} \sim \frac{\sqrt{G M r_{\mathrm{m}}}}{2 \pi 0.4 M R^{2}} \dot{M} \sim \frac{G^{3 / 7} \mu^{2 / 7} \dot{M}^{6 / 7}}{2 \pi 0.4 M^{4 / 7} R^{2}}$

$\dot{\nu} \sim 3.5 \times 10^{-13} \mu_{30}^{2 / 7}\left(\frac{\dot{M}}{10^{16} \mathrm{~g} \mathrm{~s}^{-1}}\right)^{6 / 7} \mathrm{~s}^{-2}$

$\dot{\nu} \sim 2.5 \times 10^{-13} \mu_{30}^{2 / 7}\left(\frac{L_{\mathrm{X}}}{10^{36} \mathrm{erg} \mathrm{s}^{-1}}\right)^{6 / 7} \mathrm{~s}^{-2}$

The time scale $t_{\mathrm{su}}=\nu / \dot{\nu}$ for spinning-up the accreting NS is

$t_{\mathrm{su}} \sim 3 \times 10^{5} \nu^{4 / 3}\left(\frac{10^{37} \mathrm{erg} \mathrm{s}^{-1}}{\mathrm{~L}_{\mathrm{X}}}\right)\left(\frac{r_{\mathrm{co}}}{r_{\mathrm{m}}}\right)^{1 / 2} \mathrm{yr}$
For typical mass accretion rates occurring in highluminosity sources $\left(10^{37}-10^{38} \mathrm{erg} \mathrm{s}^{-1}\right)$, this time scale is short and spin-up should be clearly observable. Indeed, the spin-up of Cen X-3 was already revealed since its first observations (see Bildsten et al. 1997 for a compilation of spin-period time histories of several accretion-powered pulsars).

To illustrate the dependence of spin-up rate on $\mathrm{X}$ ray luminosity outlined in eq. 10 , let us consider the case of A0535+262, one of the brightest HMXB transients. In this system the NS accretes from its Betype companion via a thin disk. The mass accretion is not continuous, but it occurs via rare violent outbursts, during which the accretion rate changes by more than three orders of magnitude, resulting in luminosities from $(0.5-1) \times 10^{34}$ to $\sim 10^{38} \mathrm{erg} \mathrm{s}^{-1}$. The dependence of its spin-up rate on the X-ray luminosity gives us a direct probe of the model of diskmagnetosphere interaction and of the properties of the magnetosphere. When A0535+262 is at high luminosity, the accretion disk squeezes the magnetosphere to sizes much smaller than the corotation radius, thus reducing the flow-magnetosphere interaction to the simple scenario outlined above. Fig 4 shows how the observed spin-up rate depends on the X-ray flux, as observed during the bright phase of an outburst which occurred in 1996 (Bildsten et al. 1997). The dotted line indicates the relation $\dot{\nu} \propto \dot{M}^{6 / 7}$, deduced from the simple model described above. The observed points closely follow the model prediction and yield a NS surface magnetic field $B \sim 10^{12} \mathrm{G}$, which roughly agrees with the value derived from studies of cyclotron absorption lines.

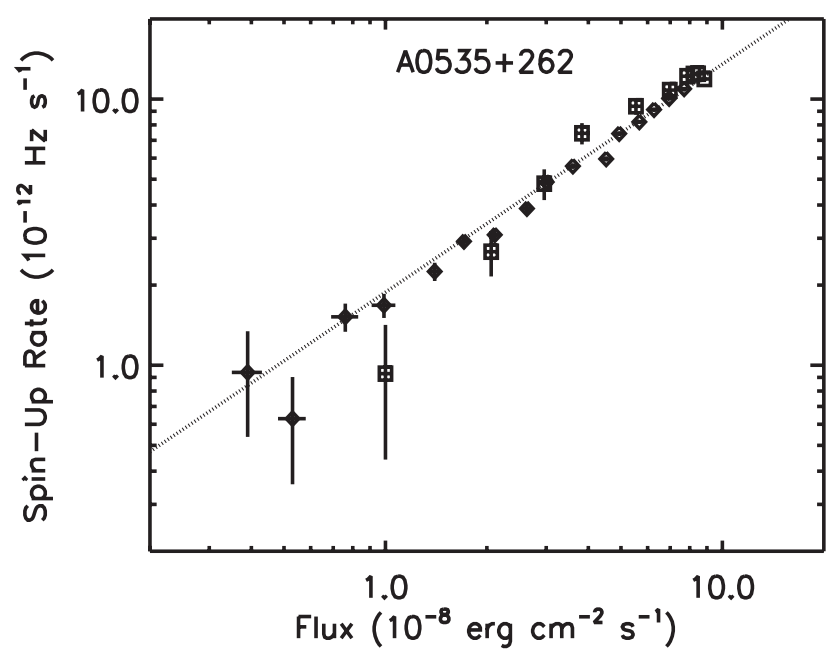

Fig. 4 Dependence of NS spin-up rate on X-ray flux (i.e. mass accretion rate). The dotted line indicates the prediction of the simple model of interaction of a dipole magnetosphere with a Keplerian disk (from Bildsten et al. 1997). 
A complete description of the spin-up/spin-down behaviour of accreting NSs is much more complicated than the simple picture outlined above, owing to the interaction between the accretion flow and the magnetosphere, which is particularly complex when the NS is rotating close to the equilibrium period, i.e. when $r_{\mathrm{m}} \approx r_{\mathrm{co}}$, and in the propeller stage, when $r_{\mathrm{m}}>r_{\mathrm{co}}$. These difficulties are illustrated, e.g., by the case of KS $1947+300$, for which the magnetic field derived from the $\dot{\nu}-L_{\mathrm{X}}$ correlation (Tsygankov \& Lutovinov 2005) is more than one order of magnitude higher than that indicated by the cyclotron line detected in this source.
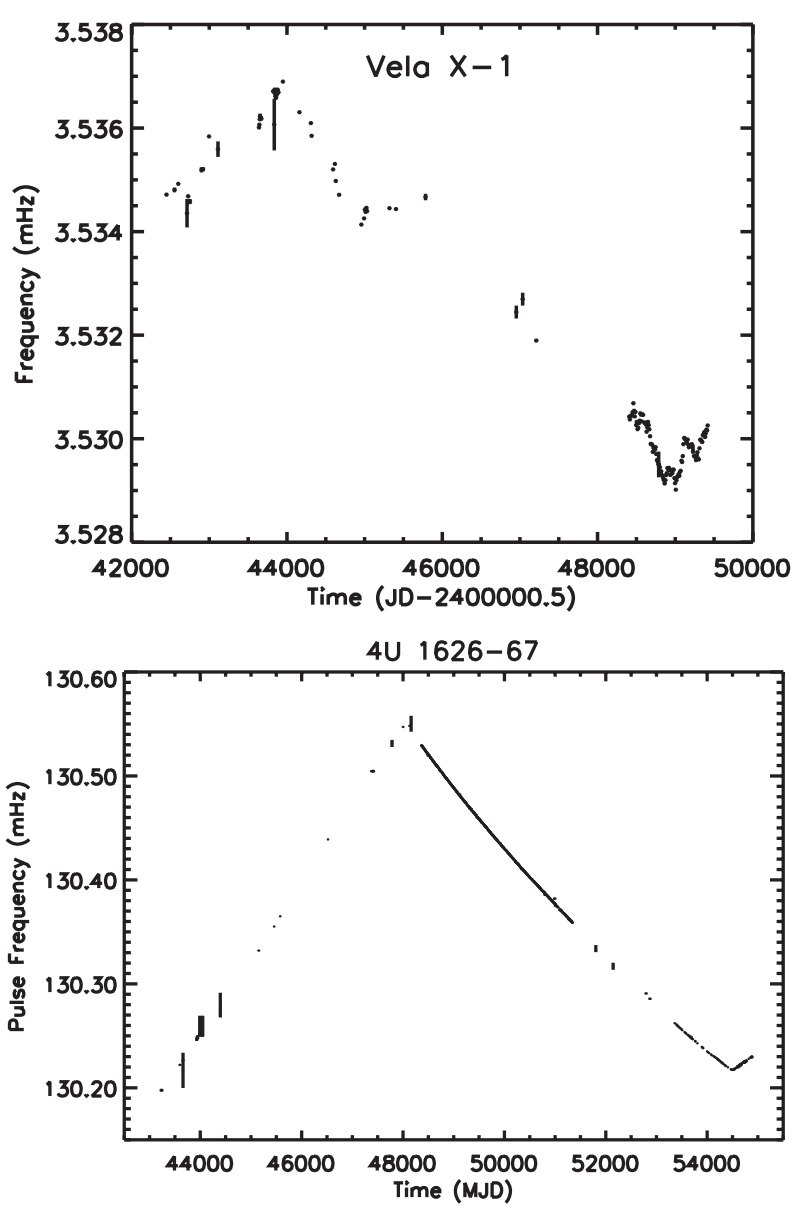

Fig. 5 Examples of spin-up/spin-down transitions of the wind accreting magnetic NS in Vela X-1 (top panel, from Bildsten et al. 1997) and the disk accreting magnetic NS in 4U 1626-67 (bottom panel, from Camero-Arranz et al. 2010).

The existence of counteracting torques (eq. 99) implies that: 1) there should be time periods of torque reversal, and 2) NSs should have some equilibrium spin periods. Indeed, observations do demonstrate time periods with zero time derivative of the NS spin value in some disk-accreting systems (e.g. Parmar et al. 1989,
Wilson et al. 2002: Baykal et al. 2002). Alternating periods of spin-up and spin-down are common in windaccreting systems, where the angular momentum of the gravitationally-captured matter is subject to large fluctuations of both signs (see, e.g., the spin history of the wind accreting NS in Vela X-1 in the upper panel of Fig 5), but they have also been observed in NSs which are known to accrete through a disk (an example is shown in the bottom panel of Fig 57. A comprehensive review of observational facts about spin-up and spindown histories can be found in, e.g., Bildsten et al. (1997).

In these cases the observed luminosity/mass accretion rate values were used to make an estimate of the NS magnetic field, adopting some prescription of the spin-down torque $K_{\mathrm{sd}}$. Application of this approach to A $0535+262$ provides a NS magnetic field value in agreement with that known from position of cyclotron absorption line (Doroshenko et al. 2014). The problem of torque reversals in wind-accreting (without Keplerian accretion disk) binaries has been recently re-examined in the context of the quasi-spherical subsonic accretion regime by (Shakura et al. 2012). Application of this model to Vela X-1 and GX 301-2 allowed these authors to estimate magnetic fields consistent with those derived from the cyclotron lines.

\section{Aperiodic X-ray flux variations as a tool to measure NS magnetospheres}

The availability of high-quality data on accreting X-ray binaries has made it possible to investigate the NS magnetospheres using new methods based on the study of their aperiodic X-ray variability. Aperiodic variability in the X-ray flux of accreting sources was discovered at the dawn of X-ray astronomy (e.g., Oda et al.|1971) and since then it has been extensively studied, mostly in the frequency domain, through the use of Fourier analysis techniques (van der Klis 1989). The noise power spectrum in accreting sources has been shown to follow a power law in broad range of frequencies.

It is now widely accepted that this variability originates from the modulation of the instantaneous values of the mass accretion rate at different distances from the central object and their subsequent multiplicative superposition (so called model of propagating fluctuations; Lyubarskii 1997: Churazov et al. 2001; Arévalo \& Uttley 2006 Revnivtsev 2008). In the framework of this model, the varying luminosity originates in the central parts of the accretion flow (i.e. the accretion column in the case of X-ray pulsars), but the modulations of the mass accretion rate are inserted into the flow at different (including large) distances from the central object, 


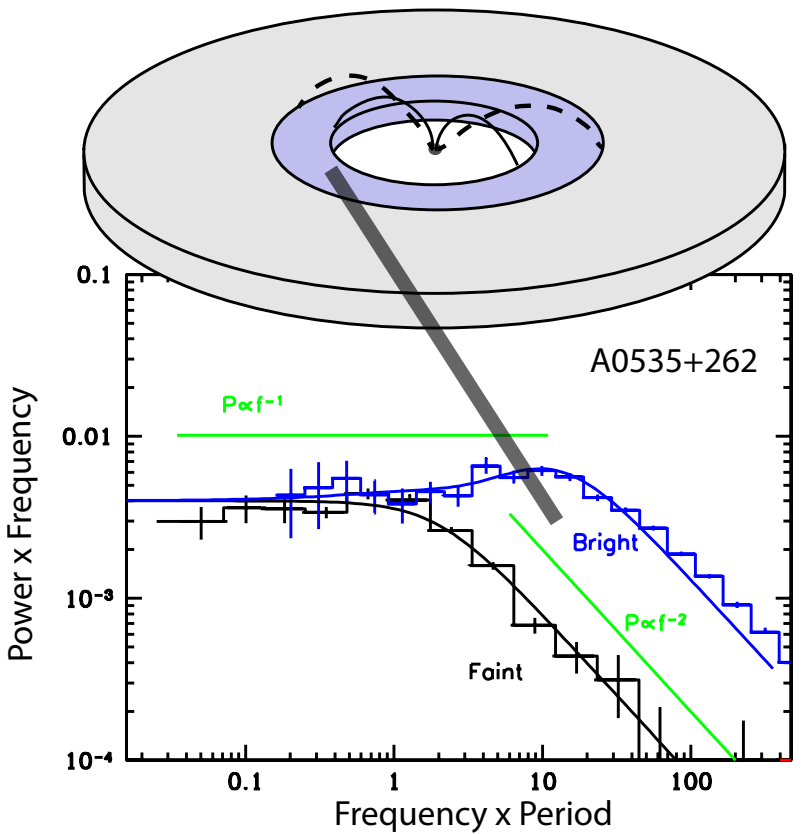

Fig. 6 Upper part: scheme of the accretion disk around a NS magnetosphere at two levels of the mass accretion rate. At high accretion rate the inner radius of the disk shrinks and an additional part of the flow with the fastest noise appears. Lower part: power spectra (pulse variations removed) of the accreting X-ray pulsar A0535+262 at different X-ray luminosities. The thick line relates the inner part of the disk with the fastest variability, not present during low accretion rate periods, when the magnetosphere pushes the disk further away from the NS.

as a result of the stochastic nature of the viscosity in accretion disks (see, e.g., Balbus \& Hawley 1991; Armitage \& Reynolds 2003, Hirose et al. 2006).

The fastest variations, originating closer to the compact object, modulate the accretion rate in the disk, which is incoming to these regions from the outer disk regions. This model predicts that, if the accretion disk is truncated at some distance from the compact object, the broad band power spectrum of its luminosity variations should show a break (or steepening) resulting from the lack of variability at the highest frequencies (see Fig 6). The break frequency, $f_{\mathrm{b}}$, should correspond to the time scale at which the inner part of the accretion flow modulates the mass accretion rate. As the truncation radius moves inward, $f_{\mathrm{b}}$ should increase. Observational support of this prediction was demonstrated by Revnivtsev et al. (2009).

If the characteristic frequency $f_{0}$ of the noise at the magnetospheric boundary $r_{\mathrm{m}}$ is proportional to the frequency of the Keplerian rotation $\nu_{\mathrm{K}}$ of matter at the inner edge of the accretion disk $r_{\text {in }} \approx r_{\mathrm{m}}$, we can relate the observed break frequency to the instantaneous value of the mass accretion rate $\dot{M}$ :

$2 \pi \nu_{\mathrm{K}}=(G M)^{1 / 2} r_{\mathrm{m}}^{-3 / 2}$

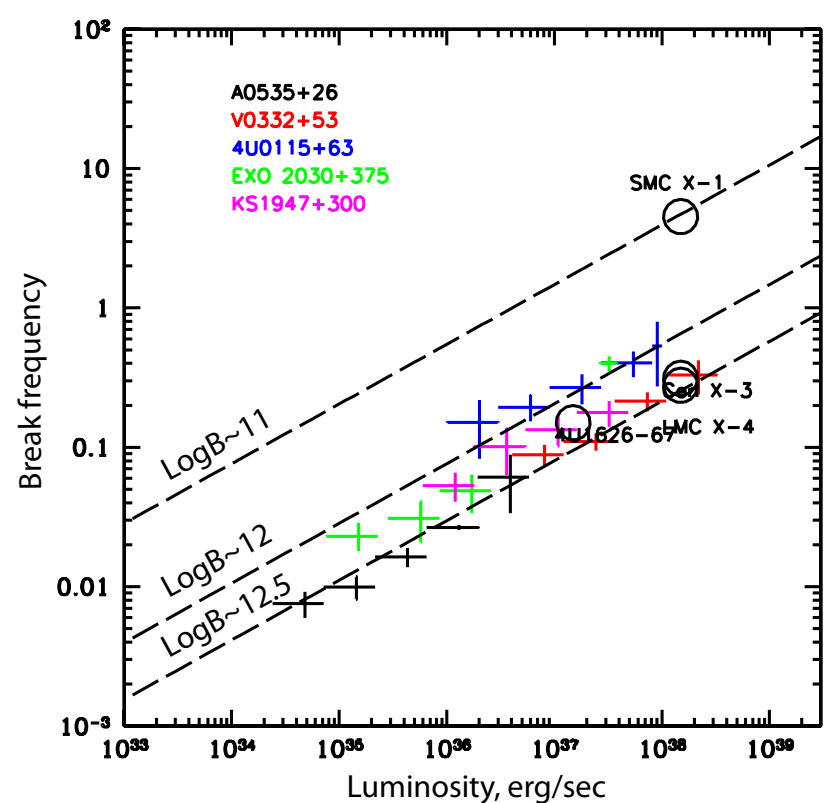

Fig. 7 Luminosity dependence of the break frequency in power spectra of disk accreting X-ray pulsars. Dashed lines denote approximate positions of breaks predicted by a simple model of Keplerian disk-dipole magnetosphere interaction with different strengths of NS surface magnetic field. The exact position of these lines should be treated with caution due to uncertainties of NS parameters and properties of the magnetosphere.

and the break frequency will follow the dependence:

$f_{\mathrm{b}} \propto f_{0} \propto \nu_{\mathrm{K}}\left(r_{\mathrm{in}}\right) \propto(G M)^{5 / 7} \mu^{-6 / 7} \dot{M}^{3 / 7}$

This relation was observed in transiently accreting pulsars (see e.g. Revnivtsev et al. 2009, Tsygankov et al. 2012, Doroshenko et al. 2014, and Fig 7).

In addition to the broad band noise, accreting Xray pulsars sometimes show quasi-periodic oscillations (QPO) of their fluxes (see e.g. Shinoda et al. 1990. Finger et al. 1996, Camero-Arranz et al. 2012a). It was found that the QPO frequency varies during the outbursts of the transient A0535+262 and its value strongly depends on the X-ray flux. It is remarkable that the dependence of the QPO frequency on the accretion rate is very similar to that of the frequency of the break in the power spectrum of the source flux variability, which hints on their common origin. It was proposed that the QPO might originate at the inner boundary of the accretion disk, truncated by the NS magnetosphere at the beat frequency between the Keplerian frequency at disk inner edge and the neutron star rotation frequency (Finger et al. 1996). 


\section{Magnetic propeller effect}

Due to the increase of magnetospheric radius with the decrease of the mass accretion rate (eq. (4)), at some stage of transiently accreting NSs (e.g. in Be HMXBs during outbursts or in LMXB transients) the magnetospheric radius can reach the corotation radius and stop the direct accretion regime. This is the so called "propeller effect". The limiting mass accretion rate $\dot{M}$, and the corresponding luminosity, can be estimated by the condition $r_{\mathrm{m}} \sim r_{\mathrm{co}}$ :

$\dot{M} \sim \xi^{7 / 2} \frac{(2 \pi)^{7 / 3} \mu^{2}}{G^{5 / 3} M^{5 / 3} P^{7 / 3}}$

$L_{\mathrm{x}} \sim \xi^{7 / 2} 2.3 \times 10^{33} \mu_{30}^{2}\left(\frac{100 \mathrm{~s}}{\mathrm{P}}\right)^{7 / 3} \quad \mathrm{erg} \mathrm{s}^{-1}$

Below we consider several consequences of the propeller effect which can be probed with observations.

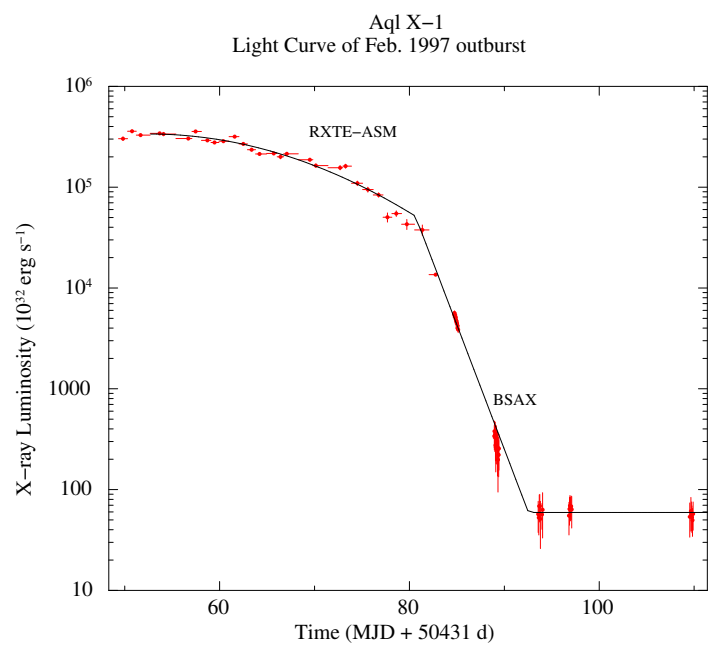

Fig. 8 Light curve of the 1997 outburst of the LMXB transient Aql X-1. After a relatively smooth decline, an abrupt drop of X-ray luminosity can be seen around $L_{\mathrm{x}} \sim 5 \times 10^{36}$ $\mathrm{erg} \mathrm{s}^{-1}$. This behavior can be explained by the the propeller effect of a rotating magnetosphere with $B \sim(1-4) \times 10^{8} \mathrm{G}$ at the NS surface (from Campana et al. 2014).

\subsection{Luminosity drops in HMXBs and LMXBs}

When the magnetospheric radius starts to exceed the corotation radius, one should expect to see an abrupt drop of the source luminosity or a switch to unsteady accretion with large luminosity swings. Indeed, at this stage the matter gains angular momentum from the rotating magnetosphere and can not move directly toward the NS. Relatively small variations of the incoming mass rate can lead to large variations of the X-ray luminosity.
Such drops were indeed observed during the decreasing phases of the oubursts of several Be binaries, like, e.g. V0332+53 (Stella et al. 1986), 4U0115+63 (Campana et al. 2001), Vela X-1 (Doroshenko et al. 2011), 4U1907+09 (Doroshenko et al. 2012a). This effect is not easy to catch because it occurs in a short time interval, when the source is crossing the above luminosity limit. An additional complication comes from the fact that when XRBs reach low luminosity levels $\left(L_{\mathrm{x}}<10^{33}-10^{34} \mathrm{erg} \mathrm{s}^{-1}\right)$, the contribution provided by X-ray emission of the companion star may become non-negligible.

It is possible that the pure propeller effect, causing the complete ejection of gravitationally captured matter, is unlikely to be realized unless the compact magnetized rotator is really fast (as in the case of the rapidly rotating magnetized white dwarf in the binary AE Aqr, see, e.g., Wynn et al. 1997). In the case of slow rotators some residual accretion might still be possible from a quasi-spherical reservoir above the magnetosphere (see e.g. Shakura et al. 2012) or from so-called "dead-disks" (Illarionov \& Sunyaev 1975, D'Angelo \& Spruit 2010 2012).

If the mass supply rate at the magnetospheric boundary persists for some time, one might expect large amplitude quasi-cyclic variations of X-ray luminosity, due to intermittent matter penetration (Baan 1979 D'Angelo \& Spruit 2010, 2012). Such variations were observed in several cases: A0535+262 (Caballero et al. 2008; Postnov et al. 2008), EXO 2030+375 (Klochkov et al. 2011).

The influence of the propeller effect has also been observed in transiently accreting LMXBs (Fig 8), where abrupt drops of X-ray luminosity were seen in the deacying parts of the outbursts (see, e.g., Cui 1997, Gilfanov et al. 1998; Asai et al. 2013; Campana et al. 2014), leading to estimated magnetic fields of a few $10^{8} \mathrm{G}$. Note, that in the case of fast pulsars in LMXBs the inner edge of the disk is much closer to the NS surface than in HMXBs. Thus the luminosity variations associated to the propeller effect are smaller and more difficult to detect. Also considering that the determination of $r_{\mathrm{m}}$ is subject to larger uncertainties in these systems, the above estimates should be taken with some caution (see, e.g., discussion in Patruno \& Watts 2012).

\subsection{Influence of the propeller effect on XRBs populations}

The presence of rotating NS magnetospheres should prevent persistent accretion at low rates (see, e.g., discussion of this point in Lipunov 1982, Stella et al. 1986 
Shtykovskiy \& Gilfanov 2005 numerical simulation predict similar effect in LMXB populations, see, e.g., $\mathrm{Ku}-$ ranov et al. 2014). In principle, this prediction could be tested with statistical analysis of well defined samples of XRBs, which should reveal a lack of objects in some regions of parameters (e.g. luminosity, spin-period, magnetic field, orbital period). However, such studies are complicated by observational biases and by large uncertainties on many parameters.

Attempts to obtain a well defined sample of X-ray binaries with the help of sky surveys can be traced back to the first X-ray sky surveys (see e.g. Matilsky et al. 1973). However, it took a long time to measure the relevant physical parameters of these binaries (orbital period, distance, etc...), which are needed for quantitative comparisons with the predictions of population synthesis models.

The simplest comparison between observations and population synthesis can be done for the distribution of X-ray luminosities. This is more difficult do for the sources in our Galaxy, due to the uncertainties on their distances, but it is much easier for sources in the Magellanic Clouds. Shtykovskiy \& Gilfanov (2005) have shown that the flattening below $L_{\mathrm{x}}<10^{35} \mathrm{erg} \mathrm{s}^{-1}$ of the luminosity function of LMC X-ray sources might indicate an influence of the propeller effect. More solid confirmation of this effect is required on much higher statistics of sources at such low luminosities.

Population studies can also be used to test some of the models proposed to explain the SFXTs invoking the role of the rotating magnetosphere in the inhibition of accretion (e.g. Grebenev \& Sunyaev 2007; Bozzo et al. 2008). In these models the matter gradually accumulates above the magnetospheric boundary and occasionally finds its way to the NS surface, giving rise to the bright flares which characterize these sources.

A simple picture of stellar wind accretion predicts the existence of an "allowed" region in the $P_{\text {orb }}-L_{\mathrm{x}}$ parameter space, determined by the minimum orbital period of a NS with a massive companion not filling its Roche lobe, and the minimum mass accretion rate which can be supplied by such a massive star (Bhadkamkar \& Ghosh 2012, Lutovinov et al. 2013). This is based on the fact that the accretion rate onto a NSs at distance $a$ from a companion of mass $M_{2}$ depends almost only on the mass loss rate of the secondary $\dot{M}_{2}: L_{\mathrm{x}} \propto a^{-2} f\left(M_{2}\right)$. For OB stars with wind velocities $v_{\mathrm{w}} \sim 1000 \mathrm{~km} \mathrm{~s}^{-1}$, the mass loss rate $\dot{M}_{2} \propto M_{2}^{\alpha}$, with $\alpha \sim 2.76$. Therefore, for any fixed separation $a$ (i.e. approximately fixed $P_{\text {orb }}$ ), the NSs with the smallest mass companions should have the lowest X-ray luminosities. This lower boundary on $L_{\mathrm{x}}$ for persistently accreting sources should have a functional form
$L_{\mathrm{x}} \propto a^{-2} \propto P_{\mathrm{orb}}^{-4 / 3}$. If an X-ray luminosity below this limit is observed, some additional mechanism must be invoked to reduce the NS mass accretion rate.

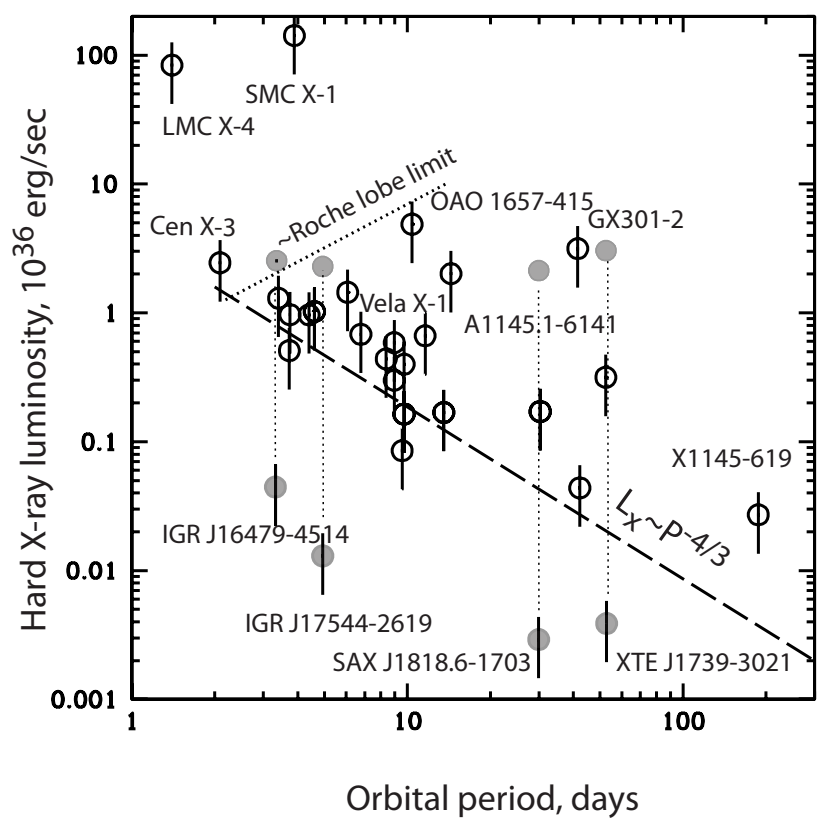

Fig. 9 Hard X-ray luminosity versus orbital period of persistent HMXBs of our Galaxy. Sources below the dotted line accrete from stellar wind, sources above this line accrete via Roche lobe overflow. The dashed line denotes the approximate lower limit of persistent X-ray luminosity which can be provided by accretion from minimally outflowing massive stars (i.e. from young stars with masses around $M_{2} \sim$ $\left.7-8 M_{\odot}\right)$. Filled circles connected with vertical dotted lines denote positions of supergiant fast X-ray transients at their average (lower circle) and maximum (upper circle) luminosity state. Adapted from Lutovinov et al. (2013).

This prediction can be tested using the currently best available flux-limited sample of accreting NSs in HMXBs presented in Lutovinov et al. (2013). This survey is complete over the whole Galaxy down to luminosities $(0.5-1) \times 10^{35} \mathrm{erg} \mathrm{s}^{-1}$ in the energy range $17-60$ $\mathrm{keV}$. As shown in Fig. 9 the persistent NSs with OB giant companions in our Galaxy populate the "allowed" area in the $P_{\mathrm{orb}}-L_{\mathrm{x}}$ diagram. On the other hand, a few sources belonging to the class of supergiant fast X-ray transients lie below the $L_{\mathrm{x}}$ lower boundary. According to above mentioned arguments, this suggests that that mass accretion onto the NS surface is inhibited (or strongly reduced) as a result of magnetospheric/centrifugal inhibition of accretion. 


\section{Conclusions}

Since the time of their discovery in the late 60's, XRBs have been used as natural laboratories for studies of matter in conditions of extreme density and magnetic fields. A substantial fraction of them contains accreting NSs endowed with magnetic fields sufficiently strong to significantly affect their observed properties. The presence of periodic X-ray pulsations provided immediate evidence for magnetic fields of the order $\sim 10^{12} \mathrm{G}$ in the HMXBs, similar to those deduced in a completely independent way from the spin-down rate of young radio pulsars. At the same time, the rarity of pulsations in LMXBs pointed to much smaller magnetic fields for the NSs contained in these, generally older, systems.

The detection of cyclotron resonance features gives the most direct way to estimate the magnetic field in XRBs. The wealth of good observational data on XRB cyclotron lines is now posing challenges to the theory developed for the spectral formation in accreting magnetized NSs, despite the high level of complexity now reached by these models. However, despite some difficulties in the detailed modelization, it is well established that the observed line energies correspond to magnetic fields in the range $\sim 10^{12}-10^{13} \mathrm{G}$.

Other ways to estimate the magnetic field in XRBs are less direct than cyclotron line measurements (and often more model-dependent), but have the advantage that they can be applied over a larger range of magnetic field intensities. The study of spin-up and/or spin-down rates in connection with the source luminosity gives the possibility to estimate the large scale magnetic field of the NS, because the exchange of angular momentum between the NS and the surrounding matter is strongly mediated by the magnetic field. The results obtained by these analysis are rather well established in the case of disk accretion during the bright outbursts of transient XRBs, spanning a large range of accretion rate values. In the case of (quasi-)spherical matter flows and/or more complex regimes (e.g. propeller, intermittent accretion, dead accretion disks, etc...), the results are subject to larger uncertainties. Other successful techniques to estimates NS magnetic field strengths rely on the observation of sudden luminosity drops due to the onset of the propeller effect and on the study of rapid aperiodic variability. The first method proved particularly valuable in the case of LMXB transients, where fields of the order of a few $10^{8} \mathrm{G}$ were derived.

In general, there is a reasonably good agreement between the magnetic fields estimated with alternative methods and those obtained from cyclotron-line measurements. However, detailed comparisons have been possible only in a limited number of cases and they of- ten rely on some poorly known quantities (e.g., the mass accretion rate, the geometry of the magnetosphere, the magnetization of the accreting plasma). These uncertainties, coupled to some poorly justified assumptions, can explain some discrepant magnetic field estimates reported in the literature. It must also be remembered that the distribution of field values derived from cyclotron lines is affected by the current instrument capabilities, which make it difficult to detect narrow spectral features at energies above a few tens of $\mathrm{keV}$ and below $\sim 1 \mathrm{keV}$. Improvements in this field will be obtained by future missions with a better energy resolution in the soft X-ray range and higher sensitivity at hard X-rays.

Acknowledgements We thank all the staff of the International Space Science Institute and the organizers of the stimulating Workshop "The Strongest Magnetic Fields in the Universe". MR acknowledges the support by grant RNF 1412-01287. SM has been supported through financial contribution from the agreement ASI-INAF I/037/12/0.

\section{References}

Arévalo, P. \& Uttley, P. 2006, MNRAS, 367, 801

Armitage, P. J. \& Reynolds, C. S. 2003, MNRAS, 341, 1041

Arons, J. \& Lea, S. M. 1976, Astrophys. J., 207, 914

Asai, K., Matsuoka, M., Mihara, T., et al. 2013, Astrophys. J., 773,117

Baan, W. A. 1979, Astrophys. J., 227, 987

Balbus, S. A. \& Hawley, J. F. 1991, Astrophys. J., 376, 214

Basko, M. M. \& Sunyaev, R. A. 1976, MNRAS, 175, 395

Baumgartner, W. H., Tueller, J., Markwardt, C. B., et al. 2013, Astrophys. J. Suppl. Ser., 207, 19

Baykal, A., Stark, M. J., \& Swank, J. H. 2002, Astrophys. J., 569,903

Becker, P. A., Klochkov, D., Schönherr, G., et al. 2012, Astron. Astrophys., 544, A123

Becker, P. A. \& Wolff, M. T. 2005, Astrophys. J. Lett., 621, L45

Becker, P. A. \& Wolff, M. T. 2007, Astrophys. J., 654, 435

Bellm, E. C., Fürst, F., Pottschmidt, K., et al. 2014, Astrophys. J., 792, 108

Bhadkamkar, H. \& Ghosh, P. 2012, Astrophys. J., 746, 22

Bhalerao, V., Romano, P., Tomsick, J., et al. 2014, ArXiv e-prints

Bhattacharya, D. \& van den Heuvel, E. P. J. 1991, Physics Reports, 203, 1

Bildsten, L., Chakrabarty, D., Chiu, J., et al. 1997, Astrophys. J. Suppl. Ser., 113, 367

Bird, A. J., Bazzano, A., Bassani, L., et al. 2010, Astrophys. J. Suppl. Ser., 186, 1

Bisnovatyi-Kogan, G. S. 1991, Astron. Astrophys., 245, 528

Bisnovatyi-Kogan, G. S. \& Komberg, B. V. 1976, Soviet Astronomy Letters, 2, 130

Bozzo, E., Falanga, M., \& Stella, L. 2008, Astrophys. J., 683, 1031

Burnard, D. J., Arons, J., \& Klein, R. I. 1991, Astrophys. J., 367,575

Caballero, I., Santangelo, A., Kretschmar, P., et al. 2008, Astron. Astrophys., 480, L17 
Caballero, I. \& Wilms, J. 2012, Memorie della Societa Astronomica Italiana, 83, 230

Camero-Arranz, A., Finger, M. H., Ikhsanov, N. R., WilsonHodge, C. A., \& Beklen, E. 2010, Astrophys. J., 708, 1500

Camero-Arranz, A., Finger, M. H., Wilson-Hodge, C. A., et al. 2012a, Astrophys. J., 754, 20

Camero-Arranz, A., Pottschmidt, K., Finger, M. H., et al. 2012b, Astron. Astrophys., 546, A40

Campana, S., Brivio, F., Degenaar, N., et al. 2014, MNRAS, 441,1984

Campana, S., Colpi, M., Mereghetti, S., Stella, L., \& Tavani, M. 1998, The Astronomy and Astrophysics Review, 8, 279

Campana, S., Gastaldello, F., Stella, L., et al. 2001, Astrophys. J., 561, 924

Campbell, C. G. 1992, Geophysical and Astrophysical Fluid Dynamics, 63, 179

Churazov, E., Gilfanov, M., \& Revnivtsev, M. 2001, MNRAS, 321,759

Clark, G. W., Woo, J. W., Nagase, F., Makishima, K., \& Sakao, T. 1990, Astrophys. J., 353, 274

Coburn, W., Heindl, W. A., Gruber, D. E., et al. 2001, Astrophys. J., 552, 738

Cui, W. 1997, Astrophys. J. Lett., 482, L163

Cumming, A. 2004, Nuclear Physics B Proceedings Supplements, 132, 435

Cusumano, G., di Salvo, T., Burderi, L., et al. 1998, Astron. Astrophys., 338, L79

D’Aì, A., Cusumano, G., La Parola, V., et al. 2011, Astron. Astrophys., 532, A73

D'Angelo, C. R. \& Spruit, H. C. 2010, MNRAS, 406, 1208

D'Angelo, C. R. \& Spruit, H. C. 2012, MNRAS, 420, 416

Davidson, K. \& Ostriker, J. P. 1973, Astrophys. J., 179, 585

Davies, R. E., Fabian, A. C., \& Pringle, J. E. 1979, MNRAS, 186,779

DeCesar, M. E., Boyd, P. T., Pottschmidt, K., et al. 2013, Astrophys. J., 762, 61

Doroshenko, V., Santangelo, A., Doroshenko, R., et al. 2014, Astron. Astrophys., 561, A96

Doroshenko, V., Santangelo, A., Ducci, L., \& Klochkov, D. 2012a, Astron. Astrophys., 548, A19

Doroshenko, V., Santangelo, A., Kreykenbohm, I., \& Doroshenko, R. 2012b, Astron. Astrophys., 540, L1

Doroshenko, V., Santangelo, A., \& Suleimanov, V. 2011, Astron. Astrophys., 529, A52

Doroshenko, V., Santangelo, A., Suleimanov, V., et al. 2010a, Astron. Astrophys., 515, A10

Doroshenko, V., Suchy, S., Santangelo, A., et al. 2010b, Astron. Astrophys., 515, L1

Ducci, L., Sidoli, L., Mereghetti, S., Paizis, A., \& Romano, P. 2009, MNRAS, 398, 2152

Filippova, E. V., Tsygankov, S. S., Lutovinov, A. A., \& Sunyaev, R. A. 2005, Astronomy Letters, 31, 729

Finger, M. H., Ikhsanov, N. R., Wilson-Hodge, C. A., \& Patel, S. K. 2010, Astrophys. J., 709, 1249

Finger, M. H., Wilson, R. B., \& Harmon, B. A. 1996, Astrophys. J., 459, 288

Frank, J., King, A., \& Raine, D. 1992, Accretion power in astrophysics.

Fürst, F., Pottschmidt, K., Wilms, J., et al. 2014a, Astrophys. J. Lett., 784, L40

Fürst, F., Pottschmidt, K., Wilms, J., et al. 2014b, Astrophys. J., 780, 133

Ghosh, P. \& Lamb, F. K. 1978, Astrophys. J. Lett., 223, L83

Ghosh, P. \& Lamb, F. K. 1979, Astrophys. J., 232, 259

Giacconi, R., Gursky, H., Kellogg, E., Schreier, E., \& Tananbaum, H. 1971, Astrophys. J. Lett., 167, L67
Giacconi, R., Gursky, H., Paolini, F. R., \& Rossi, B. B. 1962, Physical Review Letters, 9, 439

Gilfanov, M., Revnivtsev, M., Sunyaev, R., \& Churazov, E. 1998, Astron. Astrophys., 338, L83

Ginzburg, V. L. 1964, Soviet Physics Doklady, 9, 329

Gnedin, I. N. \& Sunyaev, R. A. 1974, Astron. Astrophys., 36, 379

Gold, T. 1968, Nature, 218, 731

Grebenev, S. A. \& Sunyaev, R. A. 2007, Astronomy Letters, 33, 149

Gunn, J. E. \& Ostriker, J. P. 1969, Nature, 221, 454

Harding, A. K. \& Lai, D. 2006, Reports on Progress in Physics, 69, 2631

Heindl, W., Coburn, W., Kreykenbohm, I., \& Wilms, J. 2003, The Astronomer's Telegram, 200, 1

Heindl, W. A., Coburn, W., Gruber, D. E., et al. 2001, Astrophys. J. Lett., 563, L35

Hemphill, P. B., Rothschild, R. E., Markowitz, A., et al. 2014, Astrophys. J., 792, 14

Hewish, A., Bell, S. J., Pilkington, J. D. H., Scott, P. F., \& Collins, R. A. 1968, Nature, 217, 709

Hirose, S., Krolik, J. H., \& Stone, J. M. 2006, Astrophys. J., 640,901

Ho, W. C. G., Klus, H., Coe, M. J., \& Andersson, N. 2014, MNRAS, 437, 3664

Ikhsanov, N. R. 2007, MNRAS, 375, 698

Illarionov, A. F. \& Kompaneets, D. A. 1990, MNRAS, 247, 219

Illarionov, A. F. \& Sunyaev, R. A. 1975, Astron. Astrophys., 39, 185

in't Zand, J. J. M. 2005, Astron. Astrophys., 441, L1

Kendziorra, E., Mony, B., Kretschmar, P., et al. 1992, in NASA Conference Publication, Vol. 3137, NASA Conference Publication, ed. C. R. Shrader, N. Gehrels, \& B. Dennis, 217

Klein, R. I., Arons, J., Jernigan, G., \& Hsu, J. J.-L. 1996, Astrophys. J. Lett., 457, L85

Klochkov, D., Doroshenko, V., Santangelo, A., et al. 2012, Astron. Astrophys., 542, L28

Klochkov, D., Ferrigno, C., Santangelo, A., et al. 2011, Astron. Astrophys., 536, L8

Kluźniak, W. \& Rappaport, S. 2007, Astrophys. J., 671, 1990

Kreykenbohm, I., Wilms, J., Coburn, W., et al. 2004, Astron. Astrophys., 427, 975

Krivonos, R., Tsygankov, S., Revnivtsev, M., et al. 2010, Astron. Astrophys., 523, A61

Kundt, W. 1976, Physics Letters A, 57, 195

Kuranov, A. G., Postnov, K. A., \& Revnivtsev, M. G. 2014, Astronomy Letters, 40, 29

Kuulkers, E. 2005, in American Institute of Physics Conference Series, Vol. 797, Interacting Binaries: Accretion, Evolution, and Outcomes, ed. L. Burderi, L. A. Antonelli, F. D'Antona, T. di Salvo, G. L. Israel, L. Piersanti, A. Tornambè, \& O. Straniero, 402-409

Lai, D. 2014, in European Physical Journal Web of Conferences, Vol. 64, European Physical Journal Web of Conferences, 1001

Lamb, F. K., Fabian, A. C., Pringle, J. E., \& Lamb, D. Q. 1977, Astrophys. J., 217, 197

Lamb, F. K., Pethick, C. J., \& Pines, D. 1973, Astrophys. J., 184,271

Lamers, H. J. G. L. M., van den Heuvel, E. P. J., \& Petterson, J. A. 1976, Astron. Astrophys., 49, 327

Lesur, G. \& Longaretti, P.-Y. 2009, Astron. Astrophys., 504, 309

Lewin, W. \& van der Klis, M. 2010, Compact Stellar X-ray 
Sources

Lewin, W. H. G., van Paradijs, J., \& Taam, R. E. 1993, Space Science Reviews, 62, 223

Li, X.-D. \& Wang, Z.-R. 1996, Astron. Astrophys., 307, L5

Lipunov, V. M. 1981, Soviet Astronomy, 25, 375

Lipunov, V. M. 1982, Astrophysics and Space Science, 85, 451

Lipunov, V. M., Börner, G., \& Wadhwa, R. S. 1992, Astrophysics of Neutron Stars

Liu, Q. Z., van Paradijs, J., \& van den Heuvel, E. P. J. 2006, Astron. Astrophys., 455, 1165

Liu, Q. Z., van Paradijs, J., \& van den Heuvel, E. P. J. 2007, Astron. Astrophys., 469, 807

Lovelace, R. V. E., Romanova, M. M., \& Bisnovatyi-Kogan, G. S. 1995, MNRAS, 275, 244

Lutovinov, A. A., Revnivtsev, M. G., Tsygankov, S. S., \& Krivonos, R. A. 2013, MNRAS, 431, 327

Lyubarskii, Y. E. 1997, MNRAS, 292, 679

Lyubarskii, Y. E. \& Sunyaev, R. A. 1982, Soviet Astronomy Letters, 8, 330

Lyubarskii, Y. E. \& Sunyaev, R. A. 1988, Soviet Astronomy Letters, 14, 390

Maitra, C., Paul, B., \& Naik, S. 2012, MNRAS, 420, 2307

Makishima, K. \& Mihara, T. 1992, in Frontiers Science Series, ed. Y. Tanaka \& K. Koyama, 23

Makishima, K., Mihara, T., Ishida, M., et al. 1990, Astrophys. J. Lett., 365, L59

Matilsky, T., Gursky, H., Kellogg, E., et al. 1973, Astrophys. J., 181, 753

McBride, V. A., Wilms, J., Coe, M. J., et al. 2006, Astron. Astrophys., 451, 267

McBride, V. A., Wilms, J., Kreykenbohm, I., et al. 2007, Astron. Astrophys., 470, 1065

Mereghetti, S. \& Grindlay, J. E. 1987, Astrophys. J., 312, 727

Meszaros, P. \& Nagel, W. 1985, Astrophys. J., 298, 147

Mihara, T., Makishima, K., Kamijo, S., et al. 1991, Astrophys. J. Lett., 379, L61

Müller, D., Klochkov, D., Caballero, I., \& Santangelo, A. 2013a, Astron. Astrophys., 552, A81

Müller, S., Ferrigno, C., Kühnel, M., et al. 2013b, Astron. Astrophys., 551, A6

Müller, S., Kühnel, M., Caballero, I., et al. 2012, Astron. Astrophys., 546, A125

Mushtukov, A. A., Suleimanov, V. F., Tsygankov, S. S., \& Poutanen, J. 2014, ArXiv e-prints

Nagase, F. 1989, PASJ, 41, 1

Nagel, W. 1981, Astrophys. J., 251, 288

Nakajima, M., Mihara, T., Makishima, K., \& Niko, H. 2006, Astrophys. J., 646, 1125

Nishimura, O. 2008, Astrophys. J., 672, 1127

Nishimura, O. 2014, Astrophys. J., 781, 30

Oda, M., Gorenstein, P., Gursky, H., et al. 1971, Astrophys. J. Lett., 166, L1

Orlandini, M., Dal Fiume, D., Frontera, F., et al. 1998, Astrophys. J. Lett., 500, L163

Paizis, A. \& Sidoli, L. 2014, MNRAS, 439, 3439

Parmar, A. N., White, N. E., Stella, L., Izzo, C., \& Ferri, P. 1989, Astrophys. J., 338, 359

Patruno, A. \& Watts, A. L. 2012, ArXiv e-prints

Postnov, K., Staubert, R., Santangelo, A., et al. 2008, Astron. Astrophys., 480, L21

Postnov, K. A., Shakura, N. I., Kochetkova, A. Y., \& Hjalmarsdotter, L. 2014, in European Physical Journal Web of Conferences, Vol. 64, European Physical Journal Web of Conferences, 2002

Poutanen, J., Mushtukov, A. A., Suleimanov, V. F., et al. 2013, Astrophys. J., 777, 115
Pringle, J. E. \& Rees, M. J. 1972, Astron. Astrophys., 21, 1

Reig, P. 2011, Astrophysics and Space Science, 332, 1

Reig, P., Torrejón, J. M., \& Blay, P. 2012, MNRAS, 425, 595

Revnivtsev, M. 2008, in American Institute of Physics Conference Series, Vol. 1054, American Institute of Physics Conference Series, ed. M. Axelsson, 143-153

Revnivtsev, M., Churazov, E., Postnov, K., \& Tsygankov, S. 2009, Astron. Astrophys., 507, 1211

Revnivtsev, M. G., Sazonov, S. Y., Gilfanov, M. R., \& Sunyaev, R. A. 2003, Astronomy Letters, 29, 587

Romano, P., Krimm, H. A., Palmer, D. M., et al. 2014, Astron. Astrophys., 562, A2

Santangelo, A., del Sordo, S., Segreto, A., et al. 1998, Astron. Astrophys., 340, L55

Santangelo, A., Segreto, A., Giarrusso, S., et al. 1999, Astrophys. J. Lett., 523, L85

Schönherr, G., Wilms, J., Kretschmar, P., et al. 2007, Astron. Astrophys., 472, 353

Schreier, E., Levinson, R., Gursky, H., et al. 1972, Astrophys. J. Lett., 172, L79

Semena, A., Revnivtsev, M., Buckley, A., et al. 2014, MNRAS

Semena, A. N. \& Revnivtsev, M. G. 2012, Astronomy Letters, 38,321

Sguera, V., Bazzano, A., Bird, A. J., et al. 2006, Astrophys. J., 646,452

Shakura, N., Postnov, K., Kochetkova, A., \& Hjalmarsdotter, L. 2012, MNRAS, 420, 216

Shakura, N. I., Postnov, K. A., Kochetkova, A. Y., \& Hjalmarsdotter, L. 2013, Physics Uspekhi, 56, 321

Shibanov, I. A., Zavlin, V. E., Pavlov, G. G., \& Ventura, J. 1992, Astron. Astrophys., 266, 313

Shinoda, K., Kii, T., Mitsuda, K., et al. 1990, PASJ, 42, L27

Shrader, C. R., Sutaria, F. K., Singh, K. P., \& Macomb, D. J. 1999, Astrophys. J., 512, 920

Shtykovskiy, P. \& Gilfanov, M. 2005, Astron. Astrophys., 431, 597

Shu, F., Najita, J., Ostriker, E., et al. 1994, Astrophys. J., 429,781

Sidoli, L. 2012, in Proceedings of "An INTEGRAL view of the high-energy sky (the first 10 years)" - 9th INTEGRAL Workshop and celebration of the 10th anniversary of the launch (INTEGRAL 2012). 1519 October 2012. Bibliotheque Nationale de France, Paris, France. Published online at http://pos.sissa.it/cgibin/reader/conf.cgi? confid $=176$, id. 11

Sidoli, L., Romano, P., Mereghetti, S., et al. 2007, Astron. Astrophys., 476, 1307

Srinivasan, G. 2010, New Astronomy Reviews, 54, 93

Staubert, R., Shakura, N. I., Postnov, K., et al. 2007, Astron. Astrophys., 465, L25

Stella, L., White, N. E., \& Rosner, R. 1986, Astrophys. J., 308, 669

Strohmayer, T. E. 2001, Advances in Space Research, 28, 511

Suchy, S., Pottschmidt, K., Wilms, J., et al. 2008, Astrophys. J., 675, 1487

Sunyaev, R. A. \& Shakura, N. I. 1977, Soviet Astronomy Letters, 3, 114

Taam, R. E. \& van den Heuvel, E. P. J. 1986, Astrophys. J., 305,235

Tananbaum, H., Gursky, H., Kellogg, E. M., et al. 1972, Astrophys. J. Lett., 174, L143

Tendulkar, S. P., Fürst, F., Pottschmidt, K., et al. 2014, ArXiv e-prints

Truemper, J., Pietsch, W., Reppin, C., et al. 1978, Astrophys. J. Lett., 219, L105

Tsygankov, S. S., Krivonos, R. A., \& Lutovinov, A. A. 2012, 
MNRAS, 421, 2407

Tsygankov, S. S. \& Lutovinov, A. A. 2005, Astronomy Letters, 31,88

Tsygankov, S. S., Lutovinov, A. A., Churazov, E. M., \& Sunyaev, R. A. 2006, MNRAS, 371, 19

Tsygankov, S. S., Lutovinov, A. A., \& Serber, A. V. 2010, MNRAS, 401, 1628

Uzdensky, D. A. 2004, Astrophysics and Space Science, 292, 573

van der Klis, M. 1989, in Timing Neutron Stars, ed. H. Ögelman \& E. P. J. van den Heuvel, 27

Vaughan, B. A., van der Klis, M., Wood, K. S., et al. 1994, Astrophys. J., 435, 362

Walter, R., Rodriguez, J., Foschini, L., et al. 2003, Astron. Astrophys., 411, L427

Walter, R. \& Zurita Heras, J. 2007, Astron. Astrophys., 476 , 335

Wang, Y.-M. 1995, Astrophys. J. Lett., 449, L153

Wheaton, W. A., Doty, J. P., Primini, F. A., et al. 1979, Nature, 282, 240

White, N. E., Swank, J. H., \& Holt, S. S. 1983, Astrophys. J., 270, 711

Wijnands, R. \& van der Klis, M. 1998, Nature, 394, 344

Wilson, C. A., Finger, M. H., Coe, M. J., Laycock, S., \& Fabregat, J. 2002, Astrophys. J., 570, 287

Woltjer, L. 1964, Astrophys. J., 140, 1309

Wynn, G. A., King, A. R., \& Horne, K. 1997, MNRAS, 286, 436

Yamamoto, T., Sugizaki, M., Mihara, T., et al. 2011, PASJ, 63,751

Ziolkowski, J. 1985, Acta Astronomica, 35, 185 